\title{
Dark gas in the solar neighborhood from extinction data
}

\author{
D. Paradis ${ }^{1,2}$, K. Dobashi ${ }^{3}$, T. Shimoikura ${ }^{3}$, A. Kawamura ${ }^{4}$, T. Onishi ${ }^{5}$, Y. Fukui ${ }^{4}$, and J.-P. Bernard ${ }^{1,2}$ \\ 1 Université de Toulouse, UPS-OMP, IRAP, Toulouse, France \\ e-mail: deborah.paradis@irap.omp.eu \\ 2 CNRS, IRAP, 9 Av. du Colonel Roche, BP 44346, 31028 Toulouse Cedex 4, France \\ 3 Department of Astronomy and Earth Sciences, Tokyo Gakugei University, Koganei, 184-8501 Tokyo, Japan \\ 4 Department of Astrophysics, Nagoya University, Chikusa-ku, 464-8602 Nagoya, Japan \\ 5 Department of Physical Science, Osaka Prefecture University, Gakuen 1-1, Sakai, 599-8531 Osaka, Japan
}

Received 24 December 2011 / Accepted 15 May 2012

\begin{abstract}
Context. When modeling infrared or $\gamma$-ray data as a linear combination of observed gas tracers, excess emission has been detected compared to expectations from known neutral and molecular gas traced by $\mathrm{HI}$ and $\mathrm{CO}$ measurements, respectively. This excess might correspond to additional gas component. This so-called "dark gas" (DG) has been observed in our Galaxy, as well as the Magellanic Clouds.

Aims. For the first time, we investigate the correlation between visible extinction $\left(A_{\mathrm{V}}\right)$ data and gas tracers on large scales in the solar neighborhood, to detect DG and to verify our compatibility with previous studies.

Methods. Our work focuses on both the solar neighborhood $\left(|b|>10^{\circ}\right)$, and the inner and outer Galaxy, as well as on four individual regions: Taurus, Orion, Cepheus-Polaris, and Aquila-Ophiuchus. Thanks to the recent production of an all-sky $A_{\mathrm{V}}$ map, we first perform the correlation between $A_{\mathrm{V}}$ and both $\mathrm{HI}$ and $\mathrm{CO}$ emission over the most diffuse regions (with low-to-intermediate gas column densities), to derive the optimal $\left(A_{\mathrm{V}} / N_{\mathrm{H}}\right)^{\text {ref }}$ ratio. We then iterate the analysis over the entire regions (including low and high gas column densities) to estimate the $\mathrm{CO}-$ to- $\mathrm{H}_{2}$ conversion factor, as well as the DG mass fraction.

Results. The average extinction to gas column-density ratio in the solar neighborhood is found to be $\left(A_{\mathrm{V}} / N_{\mathrm{H}}\right)^{\mathrm{ref}}=6.53 \times$ $10^{-22} \mathrm{mag} \mathrm{\textrm {cm } ^ { 2 }}$, with significant differences between the inner and outer Galaxy, of about $60 \%$. We derive an average value of the CO-to- $\mathrm{H}_{2}$ conversion factor of $X_{\mathrm{CO}}=1.67 \times 10^{20} \mathrm{H}_{2} \mathrm{~cm}^{-2} /\left(\mathrm{K} \mathrm{km} \mathrm{s}^{-1}\right)$, with significant variations between nearby clouds. In the solar neighborhood, the gas mass in the dark component is found to be $19 \%$ relative to that in the atomic component and $164 \%$ relative to the one traced by CO. These results are compatible with a recent analysis of Planck data within the uncertainties of our measurements. We estimate the ratio of dark gas to total molecular gas to be 0.62 in the solar neighborhood. The $\mathrm{HI}$-to- $\mathrm{H}_{2}$ and $\mathrm{H}_{2}$-to- $\mathrm{CO}$ transitions appear for $A_{\mathrm{V}} \simeq 0.2 \mathrm{mag}$ and $A_{\mathrm{V}} \simeq 1.5 \mathrm{mag}$, respectively, in agreement with theoretical models of dark- $\mathrm{H}_{2}$ gas.
\end{abstract}

Key words. dust, extinction - ISM: clouds - solar neighborhood

\section{Introduction}

The interstellar medium (ISM) is composed of gas and dust, where the dust component represents about $1 \%$ of the total ISM mass. Interstellar gas provides the material for star formation, making it a central part in the life cycle of matter. The gas is either atomic, molecular, or ionized and these three phases are commonly observed using the $\mathrm{HI} 21 \mathrm{~cm}$ emission, $\mathrm{CO}$ transitions, and either $\mathrm{H}_{\alpha}$ or free-free emission, respectively. Several studies have reported an emission excess in the far-infrared (FIR) with respect to the gas, that is correlated with none of the gas tracers. These excess detections were first obtained for Galactic regions (Reach et al. 1994; Meyerdierks \& Heithausen 1996). By analyzing the diffuse $\gamma$-ray emission from the ISM, Grenier et al. (2005) concluded that there is an additional gas phase in our Galaxy called "dark gas" (DG), which has a nonnegligible mass. This component has also been discovered in the Magellanic clouds (Leroy et al. 2007; Bernard et al. 2008; Roman-Duval et al. 2010).

The Planck Collaboration (2011a) obtained all-sky maps of the dust optical depth and, comparing it with the observed gas column density, constructed a map of the DG distribution covering a large fraction of the intermediate Galactic latitude sky $\left(|b|>10^{\circ}\right)$. On average, they estimated that the mass of DG is $28 \%$ of the atomic mass and $118 \%$ of the molecular gas traced by $\mathrm{CO}$ emission in the solar neighborhood. These results indicate that the DG is detected at intermediate hydrogen column densities, corresponding to extinctions values between 0.4 and $2.5 \mathrm{mag}$. The first possible explanation is that the DG could be molecular gas, which is not detected using the $\mathrm{CO}(J=1-0)$ transition. It is indeed expected that a layer of pure $\mathrm{H}_{2}$ or dissociated CO should exist around dense clouds (e.g. Wolfire et al. 2010) that would be undetectable because at temperatures below $100 \mathrm{~K}$ (which is typical of these environments), the fluxes from the $\mathrm{H}_{2}$ rotational transitions are too low. However, this layer could also be traced using other species such as $\mathrm{C}^{+}$(see Langer et al. 2010). Other possible origins were suggested by the Planck Collaboration (2011a) to explain the observed departure from linearity between the dust optical depth $(\tau)$ and the observable gas column density, namely: (1) variations in the dust/gas ratio $(\mathrm{D} / \mathrm{G}),(2)$ weak $\mathrm{CO}$ emission undetected at the sensitivity of the CO survey, (3) optically thin approximation for the $\mathrm{HI}$ emission not valid over the entire sky, and (4) formation of dust aggregates, inducing a higher dust emissivity. For hypothesis (1), the authors concluded that variations in $\mathrm{D} / \mathrm{G}$ of about $30 \%$ are unlikely in the solar neighborhood. Assumptions (2) 
and (3) were tested by performing the analysis with an upper limit in the weak $\mathrm{CO}$ emission in one case, and with a different HI spin temperature in the other case. The authors deduced that these two effects could not account for the whole excess. The last option (4) can be tested using extinction data, since dust aggregates are expected to have a higher FIR emissivity than isolated grains, but mostly unaffected optical properties in the visible and ultra-violet. Therefore, no substantial $A_{\mathrm{V}}$ excess relative to the gas column density should be observed in that case. We note that $\gamma$-ray observations would not detect any excess if the FIR excess were due to either $\mathrm{D} / \mathrm{G}$ or dust optical property variations.

The large dust particles that emit in the FIR are also responsible for the main extinction in the visible and near-infrared (NIR). Extinction data are therefore well-suited to checking for the presence of DG. An extinction map covering the whole sky was produced by Dobashi (2011) using the Two Micron All Sky Survey Point Source Catalog (2MASS PSC). This map has since been significantly improved to correct for the background-star intrinsic colors (Dobashi et al. 2012).

This study is carried out following the methodology adopted by Planck Collaboration (2011a), but using extinction instead of FIR optical depth. The use of dust optical depth requires us to determine dust temperature and therefore make assumptions about the dust emissivity shape and/or mixing effects along the line-ofsight (LOS). On the other hand, in principle, extinction directly measures dust column density. In practice however, large-scale extinction maps derived from star counts have limited accuracy and suffer from a bias inherent to the stars being intermixed with the gas, and starlight does not sample the whole LOS. In this analysis, we use the same gas tracers as in Planck Collaboration (2011a), so that the results can be compared directly.

Here, we compare the spatial distribution of the DG seen in absorption and emission, as well as the DG masses derived from the two approaches. We also wish to investigate whether the transition between the $\mathrm{HI}$ and $\mathrm{H}_{2}$ phases is consistent with that found using FIR emission. In addition, we are interested in comparing our results with theoretical models such as that of Wolfire et al. (2010).

In Sect. 2, we present the dataset used in this analysis, in Sect. 3.1 we then describe the method we applied to perform the correlations between extinction data and gas tracers. We discuss the results in Sect. 4. Finally, a summary of our findings is provided in Sect. 5.

\section{Observations}

\subsection{Extinction data}

We used color excess maps produced by Dobashi (2011) based on the 2MASS PSC (Skrutskie et al. 2006). The maps were derived using a technique named "the $X$ percentile method" (Dobashi et al. 2008, 2009), which is an extension of the wellknown NICE method introduced by Lada et al. (1994). While the standard NICE method uses the mean color of stars found in a cell set on the sky to measure the color excess by dust, the $X$ percentile method utilizes the $X$ percentile reddest star $(X=100 \%$ is the reddest) and is unaffected by the contamination of unreddened foreground stars.

The combination of the maps of the Galactic plane and both north and south high Galactic latitudes cover the whole sky with a $1^{\prime}$ grid and a varying angular resolution (from $1^{\prime}$ to $12^{\prime}$ ) because of the "adaptive grid" mapping technique used (Cambrésy 1999). This technique adjusts the resolution to ensure a constant number of stars in the cells while measuring the color excess, and maintain a flat noise level all over the sky.

Among the color excess maps presented by Dobashi (2011), we chose to compare the $E(J-H)$ map measured in the range $50<X<95 \%$ (based on his Eq. (4)) with the gas data, because this map is relatively more sensitive than other maps and has fewer defects in the regions studied in this paper $\left(|b|>10^{\circ}\right)$. However, we note that the results obtained in the following do not change significantly when using other maps computed at different $X$ values or from the other color excess available, $E\left(H-K_{\mathrm{S}}\right)$.

Here, we should note that there is a slight but systematic offset in the original color excess maps of Dobashi (2011); this arises from the rather ambiguous determination of the background star colors, i.e., the mean star colors unreddened by dust, which is needed to determine the zero point of the color excess. In general, when deriving a color excess map on large scales using the NICE method, it is virtually impossible to determine the background star colors precisely from the observed stars themselves, because the mean intrinsic star colors should vary on the sky and there is no region without dust across the Galactic plane. For simplicity, Dobashi (2011) assumed a constant mean intrinsic star color in all directions, as for other allsky color excess maps derived in a similar way, e.g., by Rowles \& Froebrich (2009); this led to a systematic offset in the final color excess map.

This varying offset is generally not large, but can cause significant problems when attempting to detect DG. To reduce these offsets, we use the new color-excess maps of Dobashi et al. (2012), who provided a correction to the original color-excess maps of Dobashi (2011) by determining the mean intrinsic star colors as a function of Galactic coordinates using the Besançon Galactic model (Robin et al. 2003), which is one of the latest Galactic stellar population synthesis model. On the basis of this model, they generated a star catalog equivalent to the 2 MASS PSC, but free from any interstellar dust, and calculated how the mean intrinsic star colors vary across the sky when applying the $X$ percentile method to the simulated star catalog. They regarded the resulting star color maps as the background for the color-excess maps derived from the 2MASS PSC.

In addition, Dobashi et al. (2012) estimated the fraction of total dust along the LOS that escapes detection by the $X$ percentile method, by simulating the effect of a diffuse dust disk on the simulated star catalog. The method is known to underestimate the diffuse dust component extending over a large region along the LOS, but it can retrieve extinction from individual well-defined dark clouds more precisely (Dobashi et al. 2009). As a result, they found that more than $80 \%$ of the total dust along the LOS should be detected in the Galactic latitude range $|b| \gtrsim 5^{\circ}$, though the effect is more severe in the lower Galactic latitude range (e.g., by $\sim 50 \%$ at $|b| \simeq 0^{\circ}$ ). They derived a map of the detection rate of the dust disk, which can be used to correct the color excess maps for this underestimation on large scales.

The color excess maps used in this work were corrected for the background star colors as well as for the underestimation of the diffuse and extended dust component. However, the corrected maps might still be erroneous close to $|b| \simeq 0^{\circ}$, especially around the Galactic center, because of the inhomogeneous detection limits in the 2MASS PSC and/or unknown stellar populations not taken into account in the Besançon Model. Therefore, when we compare the maps with the gas data in the following, we restrict ourselves to $|b|>10^{\circ}$, where these corrections for the background and the underestimation are minimized. This 


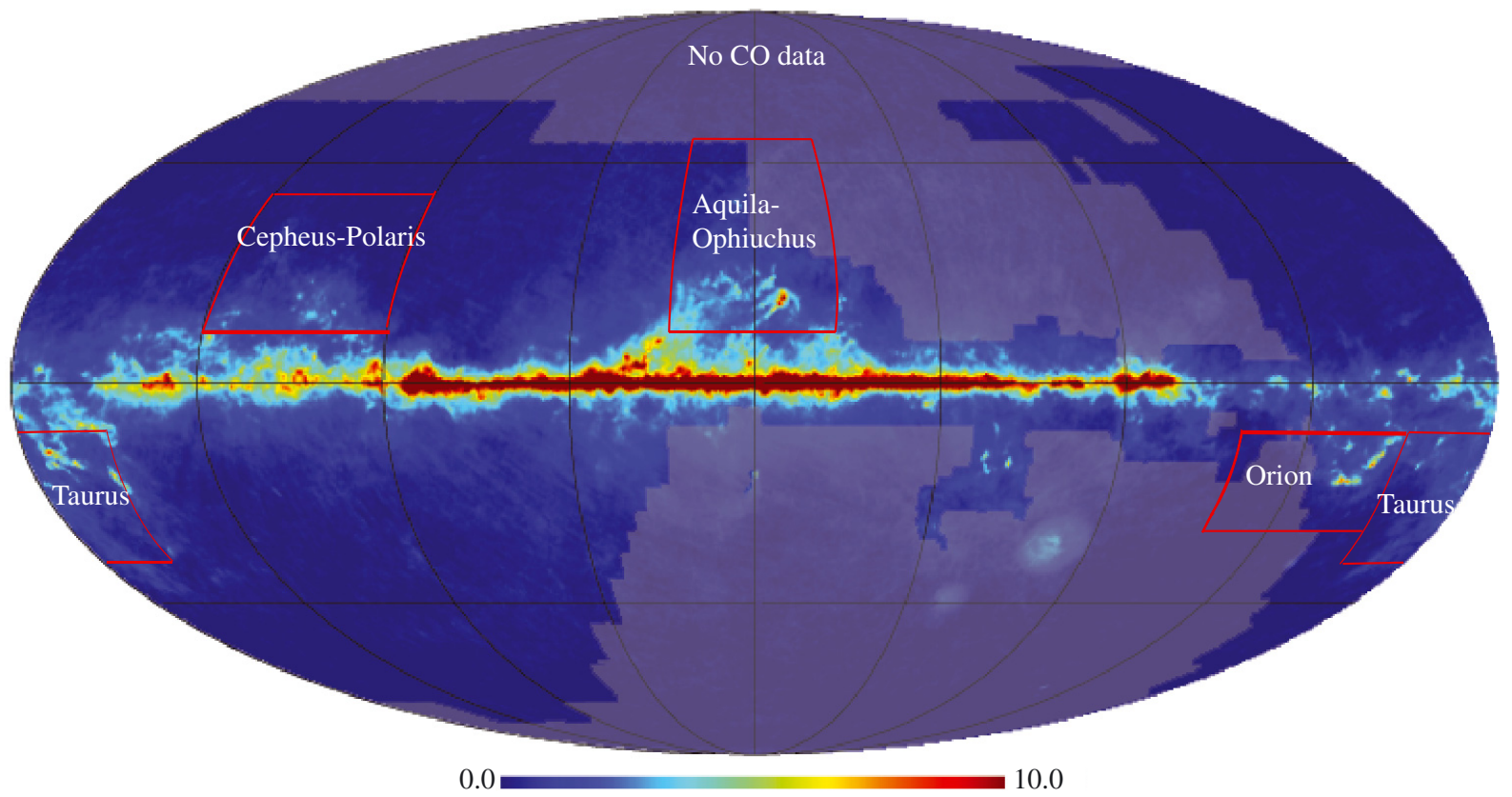

Fig. 1. Extinction map $\left(A_{\mathrm{V}}\right)$ in mag. Regions in light blue are not covered by the combination of the Dame et al. (2001) and NANTEN CO surveys, and are not used in our analyses. The red boxes correspond to individual regions defined in Sect. 3.1.3.

also corresponds to the region studied in Planck Collaboration (2011a).

Finally, we converted the $E(J-H)$ map to $A_{\mathrm{V}}$ using

$A_{\mathrm{V}}=10.9 E(J-H)$,

where the coefficient corresponds to the empirical reddening law of Cardelli et al. (1989) for $R_{\mathrm{V}}=3.1$. The resulting map (see Fig. 1) has an almost constant noise level of $\Delta A_{\mathrm{V}} \simeq 0.5 \mathrm{mag}$.

\subsection{Gas tracers}

The gas tracers used in this analysis are the same as described in Planck Collaboration (2011a). Here we provide a summary of the atomic (21 cm HI emission) and molecular $\left({ }^{12} \mathrm{CO}(J=1-0)\right.$ line) surveys. We used the LAB (Leiden/Argentine/Bonn) survey to trace the atomic gas. This survey is a combination of the Leiden/Dwingeloo survey by Hartmann \& Burton (1997) with sky observations above $-30^{\circ}$ of Galactic latitude (at a $36^{\prime}$ angular resolution), and the IAR (Instituto Argentino de Radioastronomia) survey (Arnal et al. 2000; Bajaja et al. 2005) of the Southern sky at latitudes below $-25^{\circ}$ (at a $30^{\prime}$ angular resolution). The $\mathrm{LAB}$ data were integrated in the velocity range $-400<V_{\mathrm{LSR}}<400 \mathrm{~km} \mathrm{~s}^{-1}$. Assuming that the gas is optically thin, we deduced the hydrogen column density from the integrated intensity of the $\mathrm{HI}$ emission $\left(W_{\mathrm{HI}}\right)$ using

$N_{\mathrm{HI}}=X_{\mathrm{HI}} W_{\mathrm{HI}}$,

where $X_{\mathrm{HI}}$ is the $\mathrm{HI}$ integrated intensity to column-density conversion factor. This factor is taken to be equal to $1.82 \times$ $10^{18} \mathrm{H} / \mathrm{cm}^{2} /\left(\mathrm{K} \mathrm{km} \mathrm{s}^{-1}\right)$ (Spitzer 1978).

For the molecular gas, we used the combination of three ${ }^{12} \mathrm{CO}(J=1-0)$ line surveys:

- the Dame et al. (2001) survey, in the Galactic plane, obtained with both the CfA telescope in the north (at an angular resolution of 8.4') and CfA-Chile telescope in the south (at an angular resolution of $8.8^{\prime}$ ). The integrated intensity map was derived by integrating the velocity range where the $\mathrm{CO}$ emission is significantly detected (Dame 2011);
- the unpublished high latitude survey obtained with the CfA telescope, still observing the northern sky (Dame et al., in prep.). The data cube was integrated over 10-20 velocity channels;

- the NANTEN survey obtained from Chile for the intermediate Galactic latitudes not covered by the Dame et al. (2001) survey, at a $2.6^{\prime}$ angular resolution. This survey is still unpublished, but a full description of the NANTEN telescope can be found for instance in Fukui et al. (1999). The total intensity map was obtained by integrating the data cube over the whole velocity range.

Each survey was smoothed to a common resolution of $8.8^{\prime}$ using the convolution by a Gaussian kernel. As quoted in Planck Collaboration (2011a), the NANTEN data appear to have $24 \%$ higher values than those obtained with the CfA telescopes. This discrepancy is still poorly understood and following Planck Collaboration (2011a), the NANTEN data were decreased by $24 \%$, before merging with the CfA survey, to bring them to the absolute scale of Dame et al. (2001). The total map used in this analysis is shown in Planck Collaboration (2011a), Fig. 1. The CO integrated intensity $\left(W_{\mathrm{CO}}\right)$ to column density conversion factor is derived from the relation

$N_{\mathrm{H}_{2}}=X_{\mathrm{CO}} W_{\mathrm{CO}}$.

The value of the $\mathrm{CO}$ conversion factor $\left(X_{\mathrm{CO}}\right)$ is still debated. This factor is also expected to vary over the sky. For this reason, we made no assumption and derived $X_{\mathrm{CO}}$ instead from correlations (see Sect. 3.1).

The contribution of emission in the ionized medium is neglected in this study. This assumption is reasonable since we do not consider the Galactic plane emission, which includes most of the ionized medium emission caused by HII regions. However, its possible small contribution at high latitudes, which is correlated neither with atomic nor molecular-traced gas, can be accounted for in the constant term $\left(A_{\mathrm{V}}^{0}\right)$ when performing the correlations (see Eq. (4)).

This work is done at the resolution of the HI data, i.e. $36^{\prime}$. All data are projected using the HEALPix pixelization 
Table 1. Derived parameters and their 1- $\sigma$ uncertainties, computed over different regions, for $|b|>10^{\circ}$.

\begin{tabular}{|c|c|c|c|c|c|c|c|}
\hline Region & $\begin{array}{c}\left(\frac{A_{\mathrm{V}}}{N_{\mathrm{H}}}\right)^{\mathrm{ref}} \\
\left.\left(10^{-22} \mathrm{mag} \mathrm{cm}\right)^{2}\right)\end{array}$ & $\begin{array}{c}A_{\mathrm{V}}^{0} \\
\left(10^{-2} \mathrm{mag}\right)\end{array}$ & $\begin{array}{c}X_{\mathrm{CO}} \\
\left(10^{20} \mathrm{H}_{2} \mathrm{~cm}^{-2} /\left(\mathrm{K} \mathrm{km} \mathrm{s}^{-1}\right)\right.\end{array}$ & $M_{\mathrm{H}}^{\mathrm{X}} / M_{\mathrm{H}}^{\mathrm{HI}}$ & $M_{\mathrm{H}}^{\mathrm{X}} / M_{\mathrm{H}}^{\mathrm{CO}}$ & This work & $\begin{array}{c}f_{\mathrm{DG}} \\
\text { From previous works }\end{array}$ \\
\hline All $|b|>10^{\circ}$ & $6.53 \pm 0.03$ & $6.16 \pm 0.67$ & $1.67 \pm 0.08$ & $0.19 \pm 0.25$ & $1.64 \pm 2.20$ & 0.62 & $0.55^{a}$ \\
\hline Inner Galaxy & $8.72 \pm 0.04$ & $5.81 \pm 0.16$ & $2.28 \pm 0.11$ & $0.24 \pm 0.38$ & $2.43 \pm 3.84$ & 0.71 & - \\
\hline Outer Galaxy & $5.46 \pm 0.03$ & $4.68 \pm 0.75$ & $1.67 \pm 0.10$ & $0.11 \pm 0.28$ & $0.74 \pm 1.92$ & 0.43 & - \\
\hline Cepheus-Polaris & $6.01 \pm 0.14$ & $7.93 \pm 1.33$ & $1.37 \pm 0.34$ & $0.04 \pm 0.71$ & $0.17 \pm 3.02$ & 0.15 & $0.1^{b}-0.3^{c}$ \\
\hline Taurus & $5.33 \pm 0.21$ & $0.91 \pm 0.01$ & $2.27 \pm 0.90$ & $0.42 \pm 0.02$ & $0.76 \pm 0.03$ & 0.43 & $0.3^{b}$ \\
\hline Orion & $3.41 \pm 0.11$ & $7.47 \pm 0.29$ & $2.84 \pm 0.92$ & $0.45 \pm 0.12$ & $0.66 \pm 0.18$ & 0.40 & $0.1^{b}$ \\
\hline Aquila-Ophiuchus & $6.99 \pm 0.18$ & $11.78 \pm 0.53$ & $3.46 \pm 0.91$ & $0.59 \pm 0.06$ & $1.65 \pm 0.18$ & 0.62 & $0.6^{b}$ \\
\hline
\end{tabular}

Notes. ${ }^{(a)}$ From Planck Collaboration (2011a). ${ }^{(b)}$ From Grenier et al. (2005). ${ }^{(c)}$ From Abdo, et al. (2010).

scheme (Hierarchical Equal Area isoLatitude Pixelization) ${ }^{1}$ with nside $=256$, corresponding to a pixel size of $13.7^{\prime}$. The description of the projection method used is given in Appendix A.

\section{Extinction/gas correlation}

\section{1. $\left(A_{V} / N_{H}\right)^{r e f}$ and $X_{C O}$ determination}

In Planck Collaboration (2011a), the reference value of the ratio of the dust optical depth to the gas column densities $\left(\left(\frac{\tau}{N_{\mathrm{H}}}\right)^{\text {ref }}\right)$ was determined from the correlation between $\tau$ and the gas tracers at low gas-column density. We followed the same type of analysis, and modeled the extinction $\left(A_{\mathrm{V}}^{\bmod }\right)$ in the portion of the sky covered by the infrared, atomic, and CO-traced surveys, for $|b|>10^{\circ}$, as

$A_{\mathrm{V}}^{\mathrm{mod}}=\left(\frac{A_{\mathrm{V}}}{N_{\mathrm{H}}}\right)^{\mathrm{ref}}\left(N_{\mathrm{H}}^{\mathrm{HI}}+2 X_{\mathrm{CO}} W_{\mathrm{CO}}\right)+A_{\mathrm{V}}^{0}$

where $\left(\frac{A_{\mathrm{V}}}{N_{\mathrm{H}}}\right)^{\text {ref }}$ is the reference value of the ratio of visible extinction to gas column density, and $A_{\mathrm{V}}^{0}$ is a constant, which can account for ionized gas and/or the offset in the extinction map.

However, the $A_{\mathrm{V}}$ map is particularly noisy at low column density, which makes the estimate of $\left(\frac{A_{\mathrm{V}}}{N_{\mathrm{H}}}\right)^{\text {ref }}$ difficult. We therefore adopted a slightly different approach from Planck Collaboration (2011a). Our analysis was done in two steps. We first selected regions with a limited amount of DG by identifying regions in Fig. 8 of Planck Collaboration (2011a) with DG column densities $N_{\mathrm{H}}^{\mathrm{X}}$ such that $N_{\mathrm{H}}^{\mathrm{X}} / N_{\mathrm{H}}<0.05$ and we also imposed a low $\mathrm{CO}$ content $\left(W_{\mathrm{CO}}<0.2 \mathrm{~K} \mathrm{~km} \mathrm{~s}^{-1}\right)$. For this region, which we refer to as "no DG", we derived the best-fit parameters $\left(\frac{A_{\mathrm{V}}}{N_{\mathrm{H}}}\right)^{\text {ref }}$ and $A_{\mathrm{V}}^{0}$ using Eq. (4), but did not attempt to derive $X_{\mathrm{CO}}$ in this first step because the considered regions have little $\mathrm{CO}$ emission. Second, we repeated the same analysis including all pixels of the considered region included in the gas surveys (we refered to this region as "all"), while imposing the $\left(\frac{A_{\mathrm{V}}}{N_{\mathrm{H}}}\right)^{\text {ref }}$ and $A_{\mathrm{V}}^{0}$ values derived in the first step and determined the best-fit value for $X_{\mathrm{CO}}$. We also computed the mass of DG from the difference between the best correlation and the data at that stage. The procedure above was applied to both the whole high Galactic latitude sky (see Sect. 3.1.1) and a set of individual regions (see Sect. 3.1.3), and in all cases was restricted to $|b|>10^{\circ}$.

Results of the correlations are presented in Table 1. The bestfit parameters were obtained from $\chi^{2}$ minimization. Parameter

\footnotetext{
${ }^{1}$ http://lambda.gsfc.nasa.gov/
}

uncertainties were derived from the difference between the minimum and maximum values of the parameters contained in interval $\Delta \chi^{2}$, corresponding to a confidence level of $68 \%$. We note that, for the purpose of illustrating the $A_{\mathrm{V}}$-gas correlations (see Figs. 2 and 3), we also carried the correlation in a region with $N_{\mathrm{H}}^{\mathrm{X}} / N_{\mathrm{H}}>0.05$, which we refered to as the "DG" region.

\subsubsection{High Galactic latitude regions $\left(|b|>10^{\circ}\right)$}

The correlation was first established across the whole high Galactic latitude sky $\left(|b|>10^{\circ}\right)$ characterizing the solar neighborhood. Results are displayed in Fig. 2 (top panels). We note that the linear fits (slope $=\left(\frac{A_{\mathrm{V}}}{N_{\mathrm{H}}}\right)^{\text {ref }}$ ) shown in the figures (red lines) were constrained using the whole data in the "no DG" region, and not the binned version of the data shown by yellow dots. The corresponding lines are curved as plotted on a $\log -\log$ scale, because of the constant term. The investigation of this "no DG" region leads to an $\left(A_{\mathrm{V}} / N_{\mathrm{H}}\right)^{\text {ref }}$ ratio of $6.53 \times 10^{-22} \mathrm{mag} \mathrm{cm}{ }^{2}$. This value is close to the reference value of $5.34 \times 10^{-22}$ obtained by Bohlin et al. (1978), which is relevant to the solar neighborhood and the Galactic plane. We found that the optimal $X_{\mathrm{CO}}$ is close to $1.67 \times 10^{20} \mathrm{H}_{2} \mathrm{~cm}^{-2} /\left(\mathrm{K} \mathrm{km} \mathrm{s}^{-1}\right)$. This value is lower than the findings of Planck Collaboration (2011a), who derived an averaged $X_{\mathrm{CO}}=2.54 \times 10^{20} \mathrm{H}_{2} \mathrm{~cm}^{-2} /\left(\mathrm{K} \mathrm{km} \mathrm{s}^{-1}\right)$, but in better agreement with the Galactic average of $1.9 \times$ $10^{20} \mathrm{H}_{2} \mathrm{~cm}^{-2} /\left(\mathrm{K} \mathrm{km} \mathrm{s}^{-1}\right)$ (Strong \& Mattox 1996) and the value of $1.8 \times 10^{20} \mathrm{H}_{2} \mathrm{~cm}^{-2} /\left(\mathrm{K} \mathrm{km} \mathrm{s}^{-1}\right)$ derived by Dame et al. (2001) for $|b|>5^{\circ}$.

Looking at Fig. 2, one can discern a different behavior in the $A_{\mathrm{V}}$ versus $N_{\mathrm{H}}$ plots (with $N_{\mathrm{H}}=N_{\mathrm{H}}^{\mathrm{HI}}+2 X_{\mathrm{CO}} W_{\mathrm{CO}}$ ), when comparing the "DG" and "no DG" regions at intermediate and high $A_{\mathrm{V}}$. In the first case, looking at the binned data points the correlation shows an excess between $N_{\mathrm{H}} \simeq 4 \times 10^{20} \mathrm{~cm}^{-2}\left(A_{\mathrm{V}}=0.2 \mathrm{mag}\right)$ and $N_{\mathrm{H}} \simeq 3 \times 10^{21} \mathrm{~cm}^{-2}\left(A_{\mathrm{V}}=1.5 \mathrm{mag}\right)$ compared to a linear fit, whereas we do not in the second case. We note that, owing to the low signal-to-noise ratio of the extinction data at low column densities, the location of these transitions were determined by eye here, while they were inferred from a fit in Planck Collaboration (2011a). These results imply that there is an additional gas phase, with a spatial distribution that is similar to that determined using the Planck data (Planck Collaboration 2011a). For comparison, these authors estimated that the excess appears at $A_{\mathrm{V}}$ ranging from 0.4 to $2.5 \mathrm{mag}$. However, results obtained by Planck Collaboration (2011b), by analyzing the diffuse ISM and Galactic halo, revealed some residual emission in the infrared/HI correlation, between $N_{\mathrm{H}} \simeq 3 \times 10^{20} \mathrm{~cm}^{-2}\left(A_{\mathrm{V}} \simeq 0.15 \mathrm{mag}\right)$ and $N_{\mathrm{H}} \simeq 4 \times 10^{21} \mathrm{~cm}^{-2}\left(A_{\mathrm{V}} \simeq 2.0 \mathrm{mag}\right)$. Our results are in-between these two findings based on Planck data. 

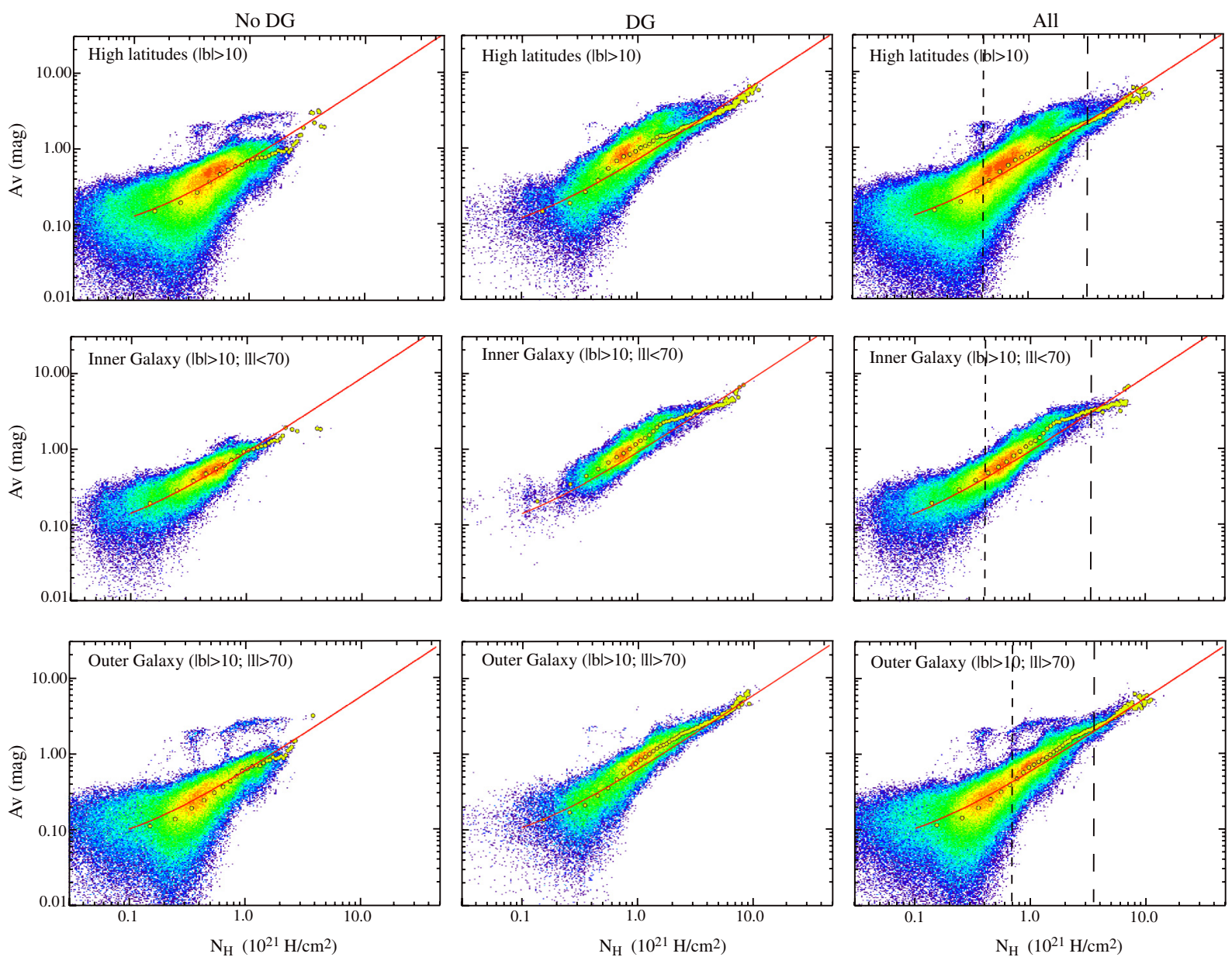

Fig. 2. Correlation plots between extinction data $\left(A_{\mathrm{V}}\right)$ and the total gas-column density $\left(N_{\mathrm{H}}=N_{\mathrm{H}}^{\mathrm{HI}}+2 X_{\mathrm{CO}} W_{\mathrm{CO}}\right)$ excluding the Galactic plane $\left(|b|>10^{\circ}\right.$ ): high latitude sky (top panels), inner Galaxy (middle panels) and outer Galaxy (bottom panels). Left, middle, and right panels correspond respectively, to regions where no DG is detected ("no DG") according to Planck Collaboration (2011a), where strong DG is detected ("DG"), and the entire region ("All") (see Sect. 3.1). The color scale represents the density of pixels on a log scale. The yellow dots show the correlation binned in $N_{\mathrm{H}}$. The red lines show the best-fit linear correlation, constrained for the whole data in the "no DG" region. The short-dashed and long-dashed lines indicate the position of a departure from a linear fit, and the position where the correlation recovers a linear behavior, respectively.

The column density of the dark component $\left(N_{\mathrm{H}}^{\mathrm{X}}\right)$ can be computed as the difference between the observed and the best-fit $\left(A_{\mathrm{V}}^{\text {mod }}\right)$ extinction per unit column density, over the entire region

$N_{\mathrm{H}}^{\mathrm{X}}=\frac{A_{\mathrm{V}}-A_{\mathrm{V}}^{\mathrm{mod}}}{\left(A_{\mathrm{V}} / N_{\mathrm{H}}\right)^{\mathrm{ref}}}$.

The total mass of hydrogen within the DG component $\left(M_{\mathrm{H}}^{\mathrm{X}}\right)$, the atomic $\left(M_{\mathrm{H}}^{\mathrm{HI}}\right)$, and CO-traced molecular gas $\left(M_{\mathrm{H}}^{\mathrm{CO}}\right)$, is estimated in the same way as in Planck Collaboration (2011a)

$M_{\mathrm{H}}^{\mathrm{X}, \mathrm{HI}, \mathrm{CO}}=m_{\mathrm{H}} D^{2} \Omega_{\text {pix }} \sum N_{\mathrm{H}}^{\mathrm{X}, \mathrm{HI}, \mathrm{CO}}$,

where $D$ is the distance to the gas, $m_{\mathrm{H}}$ the hydrogen atom mass, and $\Omega_{\text {pix }}$ the pixel solid angle. As in Planck Collaboration (2011a), we assumed the same distance to all gas components. We obtained $M_{\mathrm{H}}^{\mathrm{X}} / M_{\mathrm{H}}^{\mathrm{HI}}=0.19 \pm 0.25$ and $M_{\mathrm{H}}^{\mathrm{X}} / M_{\mathrm{H}}^{\mathrm{CO}}=1.64 \pm 2.20$. These values are expected to be both overestimated and underestimated, respectively, since the scale-height of the HI component is larger than that of the molecular one, with a probable intermediate scale-height for the DG component. The formal uncertainty in the DG mass is large and essentially results from the assumed uncertainty in $\Delta A_{\mathrm{V}} / A_{\mathrm{V}}$ and from our assumption that this uncertainty is an absolute error and therefore does not scale down with the number of independent measurements. This leads to a large uncertainty in the DG mass when most pixels in a region have low $A_{\mathrm{V}}$ values, but it is likely that our assumption about the nature of the errors overestimates the uncertainty.

Our DG mass is lower than found in Planck Collaboration (2011a), who deduced $M_{\mathrm{H}}^{\mathrm{X}} / M_{\mathrm{H}}^{\mathrm{HI}} \simeq 0.28 \pm 0.03$ and $M_{\mathrm{H}}^{\mathrm{X}} / M_{\mathrm{H}}^{\mathrm{CO}} \simeq$ $1.18 \pm 0.01$, but consistent with it, taking our large uncertainties into account. With the use of their $X_{\mathrm{CO}}$ value, we would obtain $M_{\mathrm{H}}^{\mathrm{X}} / M_{\mathrm{H}}^{\mathrm{CO}} \simeq 0.73$. Our results lead to a DG mass equal to $17 \%$ of the total observed mass, close to the value of $22 \%$ derived by Planck Collaboration (2011a). Our study using extinction data therefore reveals slightly less DG mass than the same analysis based on FIR data. The difference between the two analyses is however insignificant owing to large uncertainties for this region of the sky.

\subsubsection{Inner/outer Galaxy}

We carried out the same analysis for the inner $\left(|l|<70^{\circ}\right)$ and outer $\left(|l|>70^{\circ}\right)$ Galaxy, at high Galactic latitudes $\left(|b|>10^{\circ}\right)$. A difference appears in the $\left(A_{\mathrm{V}} / N_{\mathrm{H}}\right)^{\text {ref }}$ ratio, with a higher value being found in the inner Galaxy $\left(\left(\frac{A_{\mathrm{V}}}{N_{\mathrm{H}}}\right)^{\mathrm{ref}}=8.72 \times 10^{-22} \mathrm{mag} \mathrm{cm}^{-2}\right)$ than the outer Galaxy $\left(\left(\frac{A_{\mathrm{V}}}{N_{\mathrm{H}}}\right)^{\mathrm{ref}}=5.46 \times 10^{-22} \mathrm{mag} \mathrm{cm}^{-2}\right)$. This 

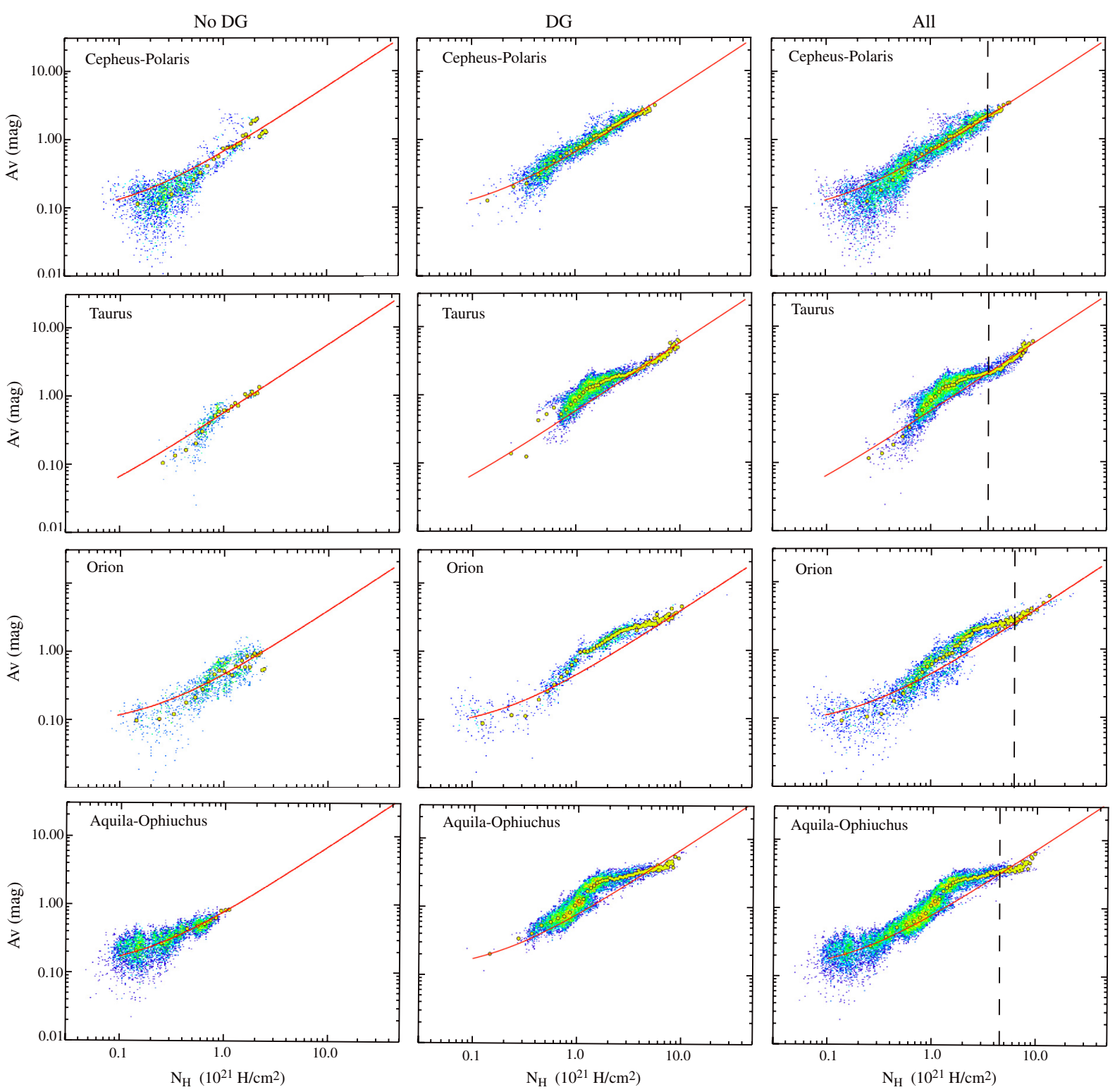

Fig. 3. Correlation plots between extinction data $\left(A_{\mathrm{V}}\right)$ and the total gas column density $\left(N_{\mathrm{H}}=N_{\mathrm{H}}^{\mathrm{HI}}+2 X_{\mathrm{CO}} W_{\mathrm{CO}}\right)$ in various regions excluding the Galactic plane $\left(|b|>10^{\circ}\right)$, from top to bottom the Cepheus-Polaris, Taurus, Orion, and Aquila-Ophiuchus regions. Left, middle, and right panels correspond respectively, to regions where no DG is detected ("no DG") according to Planck Collaboration (2011a), where strong DG is detected ("DG"), and the entire region ("All") (see Sect.3.1). The color scale represents the density of pixels on a log scale. The description of the yellow dots, red, and dashed lines is given in the caption of Fig. 2.

result could illustrate the true large-scale variations in the dust abundance and reflect the large-scale metallicity gradient in our Galaxy.

From Fig. 2, one can see that the excess is more prominent in the inner Galaxy than the outer Galaxy. In addition, we note that the outer Galaxy appears to be more noisy at low gas column densities. This is likely because the outer Galaxy hosts fewer stars than the inner Galaxy, inducing larger uncertainties in the resulting extinction map. The reconciliation between the data and the linear fit, corresponding to the $\mathrm{H}_{2}$-to- $\mathrm{CO}$ transition in the case of molecular DG, seems to occur at $N_{\mathrm{H}} \simeq 3-3.5 \times 10^{21} \mathrm{~cm}^{-2}$ $\left(A_{\mathrm{V}} \simeq 1.5-1.75 \mathrm{mag}\right)$ in both the inner and the outer Galaxy.

In term of the DG mass, each part of the Galaxy has a different behavior. The inner regions contain a more enhanced DG component, with ratios $M_{\mathrm{H}}^{\mathrm{X}} / M_{\mathrm{H}}^{\mathrm{HI}}$ and $M_{\mathrm{H}}^{\mathrm{X}} / M_{\mathrm{H}}^{\mathrm{CO}}$ of about 0.24 and 2.43, respectively. In contrast, the DG mass in the outer Galaxy represents only $11 \%$ of the gas in the atomic phase and
$74 \%$ of that in the CO-traced phase. This result indicates that the additional gas phase is essentially concentrated in the inner Galaxy. In a similar way, the $X_{\mathrm{CO}}$ factor appears to be larger in the inner Galaxy $\left(X_{\mathrm{CO}}=2.28 \times 10^{20} \mathrm{H}_{2} \mathrm{~cm}^{-2} /\left(\mathrm{K} \mathrm{km} \mathrm{s}^{-1}\right)\right)$ than its outer parts $\left(X_{\mathrm{CO}}=1.67 \times 10^{20} \mathrm{H}_{2} \mathrm{~cm}^{-2} /\left(\mathrm{K} \mathrm{km} \mathrm{s}^{-1}\right)\right)$.

\subsubsection{Individual regions}

Performing the analysis on individual regions requires the availability of enough pixels with and without DG, taking into account the limited extent of the $\mathrm{CO}$ survey. We were able to explore the $A_{\mathrm{V}}-N_{\mathrm{H}}$ correlation in the following four regions:

- Taurus $\left(200^{\circ}<l<160^{\circ} ;-35^{\circ}<b<-10^{\circ}\right)$;

- Orion $\left(240^{\circ}<l<200^{\circ} ;-30^{\circ}<b<-10^{\circ}\right)$;

- Cepheus-Polaris $\left(135^{\circ}<l<90^{\circ} ; 10^{\circ}<b<40^{\circ}\right)$;

- Aquila-Ophiuchus $\left(|l|<20^{\circ} ; 10^{\circ}<b<48^{\circ}\right)$. 
The results of the correlations for individual regions are presented in Fig. 3. Each environment has different dust properties, with $\left(\frac{A_{\mathrm{V}}}{N_{\mathrm{H}}}\right)^{\text {ref }}$ ranging between $3.41 \times 10^{-22}$ and $6.99 \times$ $10^{-22} \mathrm{mag} \mathrm{cm}^{2}$. Moreover, these regions are of great interest because they have distinctive gas properties. The $X_{\mathrm{CO}}$ factor in Orion and Aquila-Ophiuchus are larger than in the other regions. In addition, the former regions have a substantially large DG mass ratio relative to the atomic hydrogen, with ratios of 0.45 and 0.59 , respectively. These large amounts of DG in Orion and Aquila-Ophiuchus are visible in Fig. 3 as a clear, large excess in the $A_{\mathrm{V}}-N_{\mathrm{H}}$ plot. We checked that the excess was not due to contamination by YSOs. The color excess maps may indeed overestimate the true $A_{\mathrm{V}}$ if there is significant contamination by YSOs. In these two regions, we therefore also performed the correlation between extinction maps in the $J$ band $\left(A_{J}\right)$ and the gas tracers, because YSOs would induce an underestimation in the extinction maps (as opposed to the color excess maps). These results indicate that the large amount of DG is not caused by the presence of YSOs. Grenier et al. (2005) obtained a ratio $M_{\mathrm{H}}^{\mathrm{X}} / M_{\mathrm{H}}^{\mathrm{HI}}$ that is even larger than our results, in the AquilaOphiuchus-Libra region, with a ratio of 1 . In contrast, they derived a value of 0.14 in Orion. They found a ratio of 0.33 for the Taurus-Perseus-Triangulum region, close to our value of 0.42 in the Taurus region. We note however that the individual regions defined in Grenier et al. (2005) are not exactly the same as the ones used in this paper. The $A_{\mathrm{V}}-N_{\mathrm{H}}$ plot for Cepheus-Polaris does not display any noticeable excess. When comparing the DG mass with the CO one in the different clouds, we observe significant variations. We note values ranging from 0.17 in the Cepheus-Polaris region to 1.65 in Aquila-Ophiuchus, with values of 0.66 and 0.76 in Orion and Taurus. These ratios are however highly dependent on the value of the $X_{\mathrm{CO}}$ factor. The low uncertainty in the dark mass of the Taurus region is caused by the relatively large $A_{\mathrm{V}}$ values. The absolute uncertainty $\Delta A_{\mathrm{V}} / A_{\mathrm{V}}$ is larger in Cepheus-Polaris which corresponds to a large number of pixels at low $A_{\mathrm{V}}$, inducing a considerable mass uncertainty.

The $\mathrm{H}_{2}$-to-CO transition, which we determined by eye, appears at $N_{\mathrm{H}} \simeq 3.5 \times 10^{21} \mathrm{~cm}^{-2}\left(A_{\mathrm{V}} \simeq 1.75 \mathrm{mag}\right)$ in Taurus, $N_{\mathrm{H}} \simeq$ $6 \times 10^{21} \mathrm{~cm}^{-2}\left(A_{\mathrm{V}} \simeq 3.0 \mathrm{mag}\right)$ in Orion, $N_{\mathrm{H}} \simeq 3.5 \times 10^{21} \mathrm{~cm}^{-2}$ $\left(A_{\mathrm{V}} \simeq 1.75 \mathrm{mag}\right)$ in Cepheus-Polaris, and $N_{\mathrm{H}} \simeq 4 \times 10^{21} \mathrm{~cm}^{-2}$ $\left(A_{\mathrm{V}} \simeq 2.0 \mathrm{mag}\right)$ in Aquila-Ophiuchus. This transition seems to vary with environment. The position of the HI-to- $\mathrm{H}_{2}$ transition is uncertain because of the noise in the extinction data at low gascolumn densities. For this reason, we do not discuss its value further in this paper.

\section{Discussion}

The two quantities $\tau$ and $A_{\mathrm{V}}$ are in principle proportional. However, the estimate of the dust optical depth $\tau$ requires knowledge of the dust equilibrium temperature, requiring an additional step in the study of the dust/gas ratio, and additional assumptions that could bias the results. For instance, a single dust temperature is often assumed along the LOS. In addition, the temperature estimate depends on the assumption made about the emissivity spectral index $\beta$. In that sense, the use of extinction data is more straightforward. However, extinction data suffer from several defects, especially when producing the color excess map. Even if the $X$ percentile method used to generate the map is a promising method, the resulting map can still be biased by missing extinction, inducing variations in the detection rate. The detection rate, i.e., the fraction of the detected $A_{\mathrm{V}}$ to the true total $A_{\mathrm{V}}$ integrated along the LOS, could vary as a function of both the Galactic coordinates and the distance to the clouds. To determine how much extinction we should miss in the adopted $A_{\mathrm{V}}$ map, we have carried out a test by applying the $X$ percentile method to some artificial clouds set in the distribution of stars generated using the Besançon Model (Robin et al. 2003). We first prepared a spherical cloud having a Gaussian density distribution with a size of $6 \mathrm{pc}$ (at FWHM) and a total extinction along the LOS of $A_{\mathrm{V}}=10 \mathrm{mag}$ at the center of the cloud. We then located the cloud at realistic coordinates and distances of some nearby clouds in the simulated star distribution, e.g., at $(l, b)=\left(174.0^{\circ},-13.5^{\circ}\right)$ and at $D=150 \mathrm{pc}$ for the Taurus cloud, and performed the $X$ percentile method exactly in the same way as Dobashi (2011) did using the 2MASS PSC. Our results indicate that most of the individual clouds analyzed here are welldetected with a detection rate larger than $90 \%$ (i.e., the model cloud with $A_{\mathrm{V}}=10 \mathrm{mag}$ is detected as $A_{\mathrm{V}}>9$ mag), except for Orion whose detection rate is $\simeq 76 \%$. General results show that nearby clouds $(D<500 \mathrm{pc})$, which represent most of the clouds at $|b|>10^{\circ}$ in the $A_{\mathrm{V}}$ map, are clearly detected with a detection rate $>80 \%$ for all directions. The low $\left(A_{\mathrm{V}} / N_{\mathrm{H}}\right)^{\text {ref }}$ ratio derived in Orion could be the result of underestimating $A_{\mathrm{V}}$ in this region. Apart from this region, the $\left(A_{\mathrm{V}} / N_{\mathrm{H}}\right)^{\text {ref }}$ ratios in Table 1 appear equal or larger than the reference value given by Bohlin et al. (1978), confirming that the $X$ percentile method does not miss a substantial amount of extinction.

The determination of the $X_{\mathrm{CO}}$ conversion factor differs from one analysis to another. Our average value of $1.67 \times$ $10^{20} \mathrm{H}_{2} \mathrm{~cm}^{-2} /\left(\mathrm{K} \mathrm{km} \mathrm{s}^{-1}\right)$ is close to the Galactic average (Strong \& Mattox 1996) and the value derived by Dame et al. (2001) for $|b|>5^{\circ}$. A decrease in $X_{\mathrm{CO}}$ is observed from the inner to the outer high Galactic latitude sky. Most of the clouds observed in the Galactic extinction map at $|b|>10^{\circ}$ are nearby local clouds, at a distance of about $200-500$ pc, so we do not attribute the variations to the metallicity gradient (Rolleston et al. 2000), but instead to local variations. Arimoto et al. (1996), using $\mathrm{CO}$ data and virial masses, derived an average value near Sun of $2.8 \times 10^{20} \mathrm{H}_{2} \mathrm{~cm}^{-2} /\left(\mathrm{K} \mathrm{km} \mathrm{s}^{-1}\right)$, with variations ranging from $2.09 \times 10^{20}$ to $3.74 \times 10^{20} \mathrm{H}_{2} \mathrm{~cm}^{-2} /\left(\mathrm{K} \mathrm{km} \mathrm{s}^{-1}\right)$ with increasing Galactocentric radius. Abdo, et al. (2010) discovered a similar behavior, but with significant variations ranging from $0.87 \times 10^{20} \mathrm{H}_{2} \mathrm{~cm}^{-2} /\left(\mathrm{K} \mathrm{km} \mathrm{s}^{-1}\right)$ in the Gould Belt to $1.9 \times 10^{20} \mathrm{H}_{2} \mathrm{~cm}^{-2} /\left(\mathrm{K} \mathrm{km} \mathrm{s}^{-1}\right)$ in the Perseus arm. Finally, the Planck Collaboration (2011a) estimated an average value of $2.54 \times 10^{20} \mathrm{H}_{2} \mathrm{~cm}^{-2} /\left(\mathrm{K} \mathrm{km} \mathrm{s}^{-1}\right)$ in the solar neighborhood. We also note that some of the studies included the dark component in their derivation of $X_{\mathrm{CO}}$.

In their model to estimate the DG mass, Wolfire et al. (2010) predicted an $\mathrm{HI}-$ to- $\mathrm{H}_{2}$ transition located at $A_{\mathrm{V}} \simeq 0.2 \mathrm{mag}$, which agrees with our findings. This result is however slightly lower than the value derived using FIR data $\left(A_{\mathrm{V}} \simeq 0.4 \mathrm{mag}\right)$ following Planck Collaboration (2011a). However, in the latter case the HI-to- $\mathrm{H}_{2}$ transition was determined by $\chi^{2}$ minimization, whereas in our study it was done by eye, which can induce large uncertainties.

Moreover, the model predictions suggest a constant fraction of the molecular mass in the dark component of about 0.3 for an average extinction $A_{\mathrm{V}}$ around $8 \mathrm{mag}$. In cases of decreasing extinction, they found an increase in the dark mass fraction. In the framework of their model, this would indicate that a larger fraction of molecular gas is located outside the $\mathrm{CO}$ region. This fraction $\left(f_{\mathrm{DG}}\right)$ is computed as

$f_{\mathrm{DG}}=\frac{M_{\mathrm{H}}^{\mathrm{X}}}{M_{\mathrm{H}}^{\mathrm{X}}+M_{\mathrm{H}}^{\mathrm{CO}}}$. 
Values of $f_{\mathrm{DG}}$ for each region are provided in Table 1. Over the entire high Galactic latitude sky, we derived $f_{\mathrm{DG}}=0.62$, with a larger value in the inner Galaxy $\left(f_{\mathrm{DG}}=0.71\right)$ with respect to the outer Galaxy $\left(f_{\mathrm{DG}}=0.43\right)$. For comparison, using the same definition of this quantity, the Planck Collaboration (2011a) obtained a value of $f_{\mathrm{DG}} \simeq 0.55$ for the solar neighborhood. The Taurus and Aquila-Ophiuchus regions have a DG fraction of 0.43 and 0.62 , respectively. These values are in good agreement with the results obtained by Grenier et al. (2005, see Table 1) in equivalent regions, with fractions of 0.3 and 0.6 , for the Taurus and Aquila-Ophiuchus-Libra regions. These authors derived $f_{\mathrm{DG}}$ as low as 0.1 for Cepheus-Cassiopeia-Polaris and Orion, whereas Abdo, et al. (2010) deduced a value of 0.30 for Cepheus. We obtained $f_{\text {DG }}$ equal to 0.15 and 0.40 in Cepheus-Polaris and Orion.

All these studies illustrate the existence of variations in the DG mass fraction. In our study, we have excluded the Galactic plane, where the most massive and bright molecular clouds are located. At the $36^{\prime}$ angular resolution, most of the pixels considered in our analysis have extinctions lower than 10 mag. In this case, $f_{\mathrm{DG}}$ values larger than 0.3 are expected following Wolfire et al. (2010), and this is indeed what is observed here.

Dust in the form of aggregates is likely to be more emissive than isolated grains, but aggregation is not expected to substantially affect absorption properties in the visible (Kohler et al. 2011). The detection of significant departure from linearity between extinction data and gas column densities, especially in Orion or Aquila-Ophiuchus, which exhibit the largest excess in Fig. 3, indicates that dust emissivity changes induced by grain aggregation is an unlikely explanation of the observed excess. As a consequence, grain coagulation can only be responsible for a small amount of the observed excess, and may explain some of the differences in the studies based on FIR data (Planck Collaboration 2011a) and visible extinction data (this work). Moreover, our results agree with the model prediction of Wolfire et al. (2010), favoring the hypothesis of pure $\mathrm{H}_{2}$ gas (without $\mathrm{CO}$ molecules), surrounding the $\mathrm{CO}$ regions.

\section{Summary}

Using a recently revised all-sky extinction map, we have examined the correlation between the extinction and gas column densities derived from $\mathrm{HI}$ and $\mathrm{CO}$ observations. Our results can be summarized as follows:

- We have measured an excess of extinction at intermediate column densities, relative to a linear-fit between $A_{\mathrm{V}}$ and observable gas. This excess is observed over the whole high Galactic latitude sky, with a predominant amount being found toward the inner regions $\left(|l|<70^{\circ}\right)$, and in particular in Aquila-Ophiuchus. This result confirms the recent detection of DG in the Planck data and implies that the effect in the FIR is not due to changes in the dust optical properties caused by dust aggregation.

- We have derived an average dust extinction to gas ratio of $\left(A_{\mathrm{V}} / N_{\mathrm{H}}\right)^{\text {ref }}=6.53 \times 10^{-22} \mathrm{mag} \mathrm{cm}^{-2}$ in the solar neighborhood, for an average $X_{\mathrm{CO}}=1.67 \times 10^{20} \mathrm{H}_{2} \mathrm{~cm}^{-2} /\left(\mathrm{K} \mathrm{km} \mathrm{s}^{-1}\right)$. The inner/outer Galaxy exhibits a higher/lower $\left(A_{\mathrm{V}} / N_{\mathrm{H}}\right)^{\text {ref }}$ ratio $\left(8.72 \times 10^{-22} / 5.46 \times 10^{-22} \mathrm{mag} \mathrm{cm}^{-2}\right)$, associated with a higher/lower $X_{\mathrm{CO}}$ factor $\left(2.28 \times 10^{20} / 1.67 \times\right.$ $10^{20} \mathrm{H}_{2} \mathrm{~cm}^{-2} /\left(\mathrm{K} \mathrm{km} \mathrm{s}^{-1}\right)$. A significantly larger value, more than twice the value derived in the solar neighborhood is observed in the Aquila-Ophiuchus region. In contrast, the lowest $X_{\mathrm{CO}}$ value is found in Cepheus-Polaris.
- The results of our analysis agree with the theoretical predictions of modeling of the dark $\mathrm{H}_{2}$ gas obtained to help explain the observed departure from linear-fits in the correlations.

- The DG mass derived is $19 \%$ of the atomic mass and $164 \%$ of that in the CO-traced gas in the solar neighborhood. It can reach up to $59 \%$ of the atomic gas in the AquilaOphiuchus region. Our DG mass estimates are slightly lower than that derived from FIR Planck data, but the difference is insignificant.

- We have estimated the fraction of the molecular mass in the dark component $\left(f_{\mathrm{DG}}\right)$ and found an average value of 0.62 in the solar neighborhood, with variations going from 0.43 to 0.71 in the outer and inner Galaxy. We have derived $f_{\mathrm{DG}}=$ $0.15,0.43,0.40$, and 0.62 in the Cepheus-Polaris, Taurus, Orion, and Aquila-Ophiuchus regions, respectively.

- The HI-to- $\mathrm{H}_{2}$ and $\mathrm{H}_{2}$-to-CO transitions appear for $A_{\mathrm{V}} \simeq 0.2$ and $A_{\mathrm{V}} \simeq 1.5 \mathrm{mag}$ in the solar neighborhood, with variations from regions to regions.

Acknowledgements. D.P. was supported by the Centre National d'Etudes Spatiales (CNES). Part of this work was financially supported by Grant-in-Aid for Scientific Research (Nos. 22700785 and 22340040) of Japan Society for the Promotion of Science (JSPS). The authors would like to thank T. M. Dame for making the unpublished $\mathrm{CO}$ data available to them to perform this work.

\section{Appendix A: WCS to HEALPIX ancillary data transformation}

The data used in this paper were transformed from their native WCS (world coordinate system) local projection into the HEALPIx (Hierarchical Equal Area isoLatitude Pixelization) all-sky pixelization using the method described here in this Appendix. This method has also been used to produce HEALPIX maps of a larger set of ancillary data, particularly in the context of the analysis of the Planck data ${ }^{2}$. We note that the CO map used in this paper is not yet freely available, since the corresponding data have not been made public.

The HEALPIx format allows us to store ancillary data on a single grid scheme on the sphere. This is advantageous since different data can then be compared on a pixel by pixel basis, without the need to project the data to a common grid. Ancillary data are available in the WCS convention and are usually stored as Flexible Image Transport System (FITS) files. In the following, we refer to the ancillary data as FITS maps. It is usually impractical to return to the raw ancillary data and directly reprocess the maps into the HEALPIx pixelization. It is therefore necessary to project the FITS format data onto the HEALPIX pixels. As the FITS and HEALPIx pixels do not match each other in position, size, and shape, this requires some kind of interpolation, which must be done with the minimal loss of information and without altering the photometry of the original ancillary data. Here, we use a mosaicking method where we compute explicitly the surface of the pixel intersections, and use these values as weights to construct the HEALPIX map.

The HEALPIX pixels projected onto a local FITS map are shown in Fig. A.1. The calculations are performed for a HEALPIx pixel size (given by the $N_{\text {side }}$ HEALPIx parameter) so that the corresponding HEALPIx pixel size matches the Shannon criterion for the angular resolution of the data considered. This ensures that no spatial information is lost in the conversion and

2 The ancillary data can be accessed on http : //www . cesr . fr/ bernard/Ancillary/ 


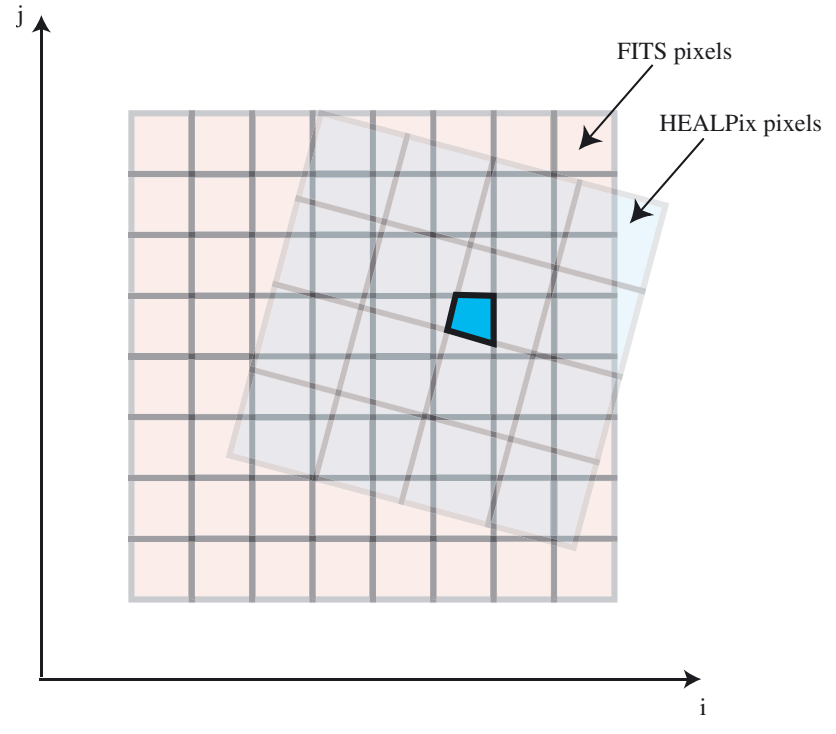

Fig. A.1. Geometry of the HEALPIx and FITS pixels.

that the corresponding HEALPIX and the FITS pixels have similar sizes. For a given FITS projection (i.e. from the astrometry information contained in the FITS header), we first identify the HEALPIx pixels that intercept the sky area covered by the FITS map. For each of those HEALPIx pixels, we identify the FITS pixels with a non-zero intersection. We then compute the surface fraction of the FITS pixels intersecting the HEALPIX pixel $S_{i h}^{i f}$, where "if" denotes the FITS pixel number and " $i h$ " denotes the HEALPIx pixel number.

The calculation is performed in the pixel space of the FITS map shown in Fig. A.1. We first compute the sky coordinates of the four corners of the considered HEALPIX pixel. We then transform these coordinates into 2D pixel numbers $(i, j)$ of the FITS image using standard routines and the astrometry information contained in the FITS header of the considered image. We then assume that the frontier of the HEALPIx pixel is a straight line in the FITS image pixel frame. Although this is not exactly true, this is a very small approximation when the size of the HEALPIX pixel is smaller or on the order of the FITS pixel size, which is always the case here. We then compute the surface of the corresponding intersection polygon, which has been normalized to that of the HEALPIx pixel surface $S_{i h}^{\text {if } 3}$.

The intersection geometry informations (if and $S_{\text {ih }}^{i f}$ ) are stored in a table containing one entry per HEALPIX pixel that has non-zero intersection with the considered FITS map. Calculations are carried and stored separately for each FITS map. We note that the above table can be easily inverted to a table with one entry per FITS pixel, giving the HEALPIx pixel numbers intercepting it $(i h)$ and the corresponding surface fraction $S_{i f}^{i h}$, which in turn can be used to perform reverse calculations projecting HEALPIX maps onto local WCS projection.

The above intersection fractions are then used to compute HEALPIX ancillary data value $d_{i h}$, given the FITS data $d_{i f}$ as

$d_{i h}=\sum_{i f} S_{i f}^{i h} \times d_{i f}$,

where the summation is carried over all FITS pixels intersecting a HEALPIx pixel $i h$.

When the data is composed of a collection of individual FITS maps, the calculation are performed for each map separately, while maintaining a map of the total weight, which allows us to evaluate average values in sky regions where individual maps overlap. We note that virtually all WCS projection types can be used, including the sixcube projection used for the COBE and FIRAS data.

\section{References}

Abdo, A. A., Ackermann, M., Ajello, M., et al. 2010, ApJ, 710, 133 Arnal, E. M., Bajaja, E., Larrarte, J. J., et al. 2000, A\&AS, 142, 35 Arimoto, N., Sofue; Y., \& Tsujimoto, T. 1996, PASJ, 48, 275

Bajaja, E., Arnal, E. M., Larrarte, E., et al. 2005, A\&A, 440, 767

Bernard, J.-P., Reach, W., Paradis, D., et al. 2008, AJ, 136, 919

Bohlin, R. C., Savage, B. D., \& Drake, F. J. 1978, ApJ, 224, 132 Cambrésy, L. 1999, A\&A, 345, 965

Cardelli, J. A., Clayton, G. C., \& Mathis, J. S. 1989, ApJ, 345, 245

Dame, T. M. 2011 [arXiv: 1101.1499]

Dame, T. M., Hartmann, D., \& Thaddeus, P. 2001, ApJ, 547, 792

Dobashi, K. 2011, PASJ, 63, S1

Dobashi, K., Bernard, J.-P., Hughes, A., et al. 2008, A\&A, 484, 205

Dobashi, K., Bernard, J.-P., Kawamura, A., et al. 2009, AJ, 137, 5099

Dobashi, K., Marshall, D. J., Shimoikura, T., \& Bernard, J.-P. 2012, PASJ, submitted

Fukui, Y., Onishi, T., Abe, R., et al. 1999, PASJ, 51, 571

Grenier, I. A., Casandjian, J. M., \& Terrier, R. 2005, Science, 307, 1292

Hartmann, D., \& Burton, W. B. 1997, Atlas of Galactic Neutral Hydrogen (Cambridge, UK: Cambridge University Press)

Kohler, M., Guillet, V., \& Jones, A. 2011, A\&A, 528, A96

Langer, W. D., Velusamy, T., Pineda, J. L., et al. 2010, A\&A, 521, A17

Lada, C. J., Lada, E. A., Clemens, D. P., \& Bally, J. 1994, ApJ, 429, 694

Leroy, A., Bolatto, A., Stanimirovic, S., et al. 2007, ApJ, 658, 1027

Meyerdierks, H., \& Heithausen, A. 1996, A\&A, 313, 929

Planck Collaboration 2011a, A\&A, 536, A17

Planck Collaboration 2011b, A\&A, 536, A24

Reach, W. T., Koo, B., \& Heiles, C. 1994, ApJ, 429, 672

Robin, A. C., Reylé, C., Derrière, S., \& Picaud, S. 2003, A\&A, 409, 523

Rolleston, W. R. J., Smartt, S. J., Dufton, P. K., \& Ryans, R. S. I. 2000, A\&A, 363,537

Roman-Duval, J., Israel, F. P., Bolatto, A., et al. 2010, A\&A, 518, A74

Rowles, J., \& Froebrich, D. 2009, MNRAS, 395, 1640

Skrutskie, M. F., Cutri, R. M., Stiening, R., et al. 2006, AJ, 131, 1163

Spitzer, L. 1978, Physical Processes in the Interstellar Medium (New-York: Wiley-Interscience)

Strong, A. W., \& Mattox, J. R., A\&A, 308, 21

Wolfire, M. G., Hollenbach, D., \& McKee, C. F. 2010, ApJ, 716, 119

\footnotetext{
3 This calculation is performed using the polyfillaa routine (see http://tir.astro.utoledo.edu/jdsmith/code/idl.php) optimized for fast clipping of polygons against a pixel grid.
} 